\title{
Correction to: There is no difference in the complication or re-operation rates between tension band wiring and locking plate fixation for olecranon fractures. The findings of a multicentre study
}

\author{
Tobenna J. Oputa $^{1}\left(\mathbb{D} \cdot\right.$ Ricci Plastow $^{1} \cdot$ James Kennedy $^{1} \cdot$ Neil Jain $^{2} \cdot$ North West Olecranon Fracture Study Group
}

Published online: 8 November 2021

๑) Springer-Verlag GmbH Germany, part of Springer Nature 2021

\section{Correction to: Archives of Orthopaedic and Trauma Surgery https://doi.org/10.1007/s00402-021-04159-6}

The original version of this article unfortunately contained a mistake. Author name Kohila Sigamoney was incorrectly written as Kohial Sigamomey in the acknowledgements section of this paper.

The correct acknowledgements section should read as:

Acknowledgements North West Olecranon Fracture Study Group: Alastair Konarski, Specialist Registrar, Trauma \& Orthopaedics, Health Education North West, ajkonarski@doctors.org.uk. Chris Jump, Specialist Registrar, Trauma \& Orthopaedics, Health Education North West, C.m.jump@doctors.org.uk. Chris Manning, Specialist Registrar, Trauma \& Orthopaedics, Health Education North West, cmanning2@doctors.org.uk. Ethan Caruana, Senior House Officer, Health Education North West, nahte14@ gmail.com. Ghazal Hodhody, Specialist Registrar, Trauma \& Orthopaedics, Health Education North West, Ghazal.

The original article can be found online at https://doi.org/10.1007/ s00402-021-04159-6.

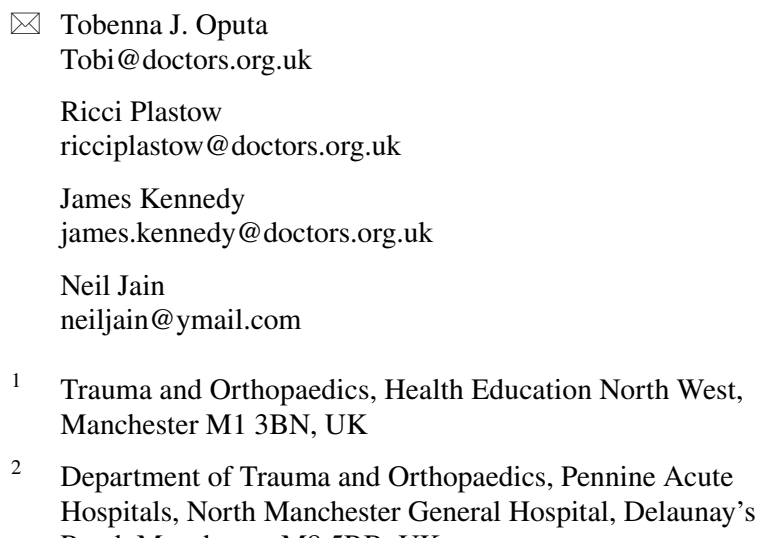

Tobenna J. Oputa

Tobi@doctors.org.uk

Ricci Plastow

ricciplastow@doctors.org.uk

James Kennedy

james.kennedy@ doctors.org.uk

Neil Jain

neiljain@ymail.com

1 Trauma and Orthopaedics, Health Education North West, Manchester M1 3BN, UK

2 Department of Trauma and Orthopaedics, Pennine Acute Hospitals, North Manchester General Hospital, Delaunay's Road, Manchester M8 5RB, UK hodhody@ doctors.org.uk. Jim Riley, Specialist Registrar, Trauma \& Orthopaedics, Health Education North West, jariley@doctors.org.uk. Johnathan Yates, Specialist Registrar, Trauma \& Orthopaedics, Health Education North West, Jonathan_yates@ hotmail.co.uk. Kai Nie, Specialist Registrar, Trauma \& Orthopaedics, Health Education North West, Kai.Nie@ nhs.net. Kenneth Koo, Specialist Registrar, Trauma \& Orthopaedics, Health Education North West, Kenneth.koo@doctors.org.uk. Kohila Sigamoney, Specialist Registrar, Trauma \& Orthopaedics, Health Education North West, Kvsorth@gmail.com. Joann Lum, Specialist Registrar, Trauma \& Orthopaedics, Health Education North West, Jlum168@msn.com. Leanne Dupley, Specialist Registrar, Trauma \& Orthopaedics, Health Education North West, leannedupley@doctors.org.uk. Sadia Afzal, Specialist Registrar, Trauma \& Orthopaedics, Health Education North West, sadia.afzal500@gmail.com. Saleem Mastan, Specialist Registrar, Trauma \& Orthopaedics, Health Education North West, S.mastan@doctors.org.uk. Tom Collins, Specialist Registrar, Trauma \& Orthopaedics, Health Education North West, tomcollins761@ gmail.com. Tom Havenhand, Senior House Officer, Trauma \& Orthopaedics, Health Education North West, tomhavenhand@ doctors.org.uk. William Fletcher, Specialist Registrar, Trauma \& Orthopaedics, Health Education North West, Fletchmk2@gmail.com.

Publisher's Note Springer Nature remains neutral with regard to jurisdictional claims in published maps and institutional affiliations. 